\title{
Aprotinin - a New Drug Candidate for The Prevention of SARS-CoV-2 (COVID-19)
}

Andrey A. Ivashchenko ${ }^{1}$, Andrey A. Svistunov ${ }^{2}$, Tatiana V. Khorobryh ${ }^{2}$, Vladimir G. Loginov ${ }^{3}$, Ruben N. Karapetian ${ }^{4}$, Natalia P. Mishchenko ${ }^{2}$, Stanislav V. Poyarkov ${ }^{5}$, Maksim V. Volgin ${ }^{2}$, Elena V. Yakubova ${ }^{4}$, Michael A. Topr ${ }^{6}$, O.V. Pyankov ${ }^{7}$, A.V. Magerramova ${ }^{7}$, A.A. Tomilov ${ }^{7}$, Alexandre V. Ivachtchenko ${ }^{8}$

Affiliations:

${ }^{1}$ ChemRar High-Tech Center, Khimki, Moscow region, Russian Federation.

${ }^{2}$ First Moscow State Medical University named I. M. Sechenov, Moscow, Russian Federation.

3 JSC "Special Economic Zones", Moscow, Russian Federation.

${ }^{4}$ Department of Biology, Chemical Diversity Research Institute, Khimki, Moscow region, Russian Federation.

${ }^{5}$ Centre for Strategic Planning of Federal Medical-Biological Agency (FMBA) Moscow, 119121, Russian Federation.

${ }^{6}$ Michael A. Topr - self employed, USA

${ }^{7}$ FBRI State Research Center of Virology and Biotechnology VECTOR, Rospotrebnadzor, Koltsovo, Novosibirsk region, Russian Federation

${ }^{8}$ Chemical Diversity Research Institute, Khimki, Moscow region, Russian Federation

Corresponding author: Ruben N. Karapetian, Department of Biology, Chemical Diversity Research Institute, Khimki, Moscow region, Russian Federation, Tel: +7-495-925-30-74 ext 572; e-mail: rk@chemrar.ru

\section{ABSTRACT}

The efficacy of Aprotinin as prophylactic treatment of Covid-19 was studied in the hamster model of SARS-CoV-2 and in the health care personnel working with Covid-19 patients in hospital. High efficacy of Aprotinin in pre- and post-exposure prophylaxis of SARS-CoV-2 was demonstrated.

Keywords: Aprotinin, prevention, SARS-CoV-2, COVID-19 


\section{INTRODUCTION}

The emergence of the new SARS-CoV-2 virus and the COVID-19 infection caused by it has posed a number of challenges for the world community. There is an intensive search for therapeutic agents around the world to prevent SARS-CoV-2 and/or treat COVID-19.

One of the important tasks is to create effective means of prophylaxis in people at high risk of infection. First of all, these are health care professionals, service, education and military personnel.

Despite active attempts to create vaccines, the majority of viable variants are expected in 2021. Note, that the world's first SARS-CoV-2 vaccine was registered in Russia on August 11, 2020 [1]. However, it takes time to evaluate the efficacy of the vaccine in preventing SARSCoV-2 without aggravating the infection, the so-called ADE phenomenon, antibody-dependent enhancement of the infection, which was observed with SARS-CoV infection in 2003. All this makes the development of effective specific preventive agents extremely urgent.

The development of preventive agents for pre- or post-exposure prophylaxis of SARSCoV-2 is equally important. Several agents have been or are being investigated in clinical trials, including lopinavir/ritonavir, emtricitabine/tenofovir alafenamide or tenofovir disoproxil fumarate, chloroquine, hydroxychloroquine, nitazoxanide, and supplements such as zinc, vitamin C, super B-complex, and vitamin D. Trials of anti-SARS-CoV-2 monoclonal antibodies and convalescent plasma are also under development. However, currently, no known agent can prevent SARS-CoV-2 infection [2].

In several studies it was shown that Aprotinin efficiently inhibits SARS-CoV-2 replication in Caco-2 (human colorectal cancer cells, [3]) and Calu-3 (human airway epithelial cells, [4]) cells in vitro. Aprotinin is a natural proteinase inhibitor obtained from bovine lung and has a long history of clinical use since 1960s. It has a broad action on proteolytic enzymes such as plasmin, trypsin, and kallikrein. Recently it was demonstrated that it inhibits also the transmembrane serine protease 2 (TMPRSS2) - the host cell protease responsible for cleavage and activation of SARS-CoV-2 S-protein [5]. TMPRSS2 is widely expressed in epithelial cells of the respiratory, gastrointestinal and urogenital tract $[6,7]$ and was shown to activate the fusion proteins of a number of respiratory viruses including human metapneumovirus, human parainfluenza viruses as well as SARS-CoV and Middle East respiratory syndrome (MERS)$\mathrm{CoV}$ in vitro $[7,8]$.

In clinical use Aprotinin is used to reduce blood loss during surgery and for pancreatitis [9, 10]. Aprotinin inhibits pro-inflammatory cytokine release and maintains glycoprotein 
homeostasis. In platelets, Aprotinin reduces glycoprotein loss (e.g., GpIb, GpIIb/IIIa), while in granulocytes it prevents the expression of pro-inflammatory adhesive glycoproteins (e.g., CD11b). Through its inhibition of multiple mediators [e.g., kallikrein, plasmin] effect of Aprotinin results in the attenuation of inflammatory responses, fibrinolysis, and thrombin generation [11]. Also Aprotinin was previously investigated against influenza viruses [12-16] and is approved for the treatment of influenza as aerosol in Russia.

All together these data demonstrates that Aprotinin has antiviral and anti-inflammatory activity. It makes Aprotinin an attractive candidate for prophylactic treatment of SARS-CoV-2 infection. To support this hypothesis we tested Aprotinin in SARS-CoV-2 hamster model. Also its potential for prophylactic treatment in human was estimated on health care personnel of Covid-hospital during 6 weeks of treatment in prospective study.

\section{METHODS}

\section{Drugs}

Gordox ${ }^{\circledR}$ (Aprotinin) $10000 \mathrm{KIU} / \mathrm{ml}$ was used in the studies.

\section{Animals}

The study was performed in the State Research Center of Virology and Biotechnology VECTOR, Koltsovo, Novosibirsk Oblast, Russia. The SARS-CoV-2 strain $\mathrm{nCov} /$ Victoria/1/2020 was used in the hamster model.

\section{SARS-CoV-2 hamster model}

Wild-type male Syrian hamsters were purchased from KrolInfor Ltd and were housed with ad libitum access to food and water. Housing conditions and experimental procedures were approved by the ethical committee of animal experimentation.

8-10 weeks old hamsters were randomized to two groups - Control and Aprotinin groups, 8 animals per group. Hamsters were anesthetized with ether and treated intranasally with sodium saline (control, $50 \mathrm{ul} /$ nostril) or Aprotinin (50 ul=500 KIU/nostril, $1000 \mathrm{KIU} /$ animal). 1 hour after drug treatment animals were inoculated with $1000 \mathrm{xCPE}_{50} /$ animal of SARS-CoV-2. The treatment of animals was performed TID and was continued during 2 days. 2-3 hours after morning treatment on the day 3 , following tissues were collected for analysis: nasal washes and lung tissues. SARS-CoV-2 RNA was measured using real-time PCR $(\mathrm{Ct}=40$ at low limit of quantitation). Weight changes in body weight of hamsters were determined at 3 day p.i.

\section{Human study}

The efficacy study of the preventive action of Aprotinin was investigated in the prospective study "Prophylaxis and prevention of Covid-infection progression" (study protocol 
№09-20 from 05.05.2020, approved by Local Ethics Committee of the I.M. Sechenov First Moscow State Medical University 07.05.2020). It was carried out in 32 health care professionals of COVID hospital of the I.M. Sechenov First Moscow State Medical University (Sechenov University) including medical and nursing staff who were constantly working in the "red zone" during three months of observation. Among them: 5 intensivists, 2 surgeons who performed tracheostomy and pleural punctures, 15 physicians and 10 nurses.

At the beginning of the study, pharmacological prevention of SARS-CoV-2 (COVID-19) in adults recommended by the Ministry of Health of the Russian Federation was used [17]. However, after the first week of administration of hydroxychloroquine as a preventive agent, health care professionals of the COVID hospital group were forced to discontinue the drug due to significant side effects. 3 weeks after the opening of the COVID hospital, employees from the COVID hospital group started Aprotinin nasal spray twice daily: in the morning, before entering the red zone and in the evening, after leaving the red zone, $400 \mathrm{KIU}$ b.i.d., totally, 800 KIU daily. As a supportive therapy, the COVID hospital staff was administered Galavit, an

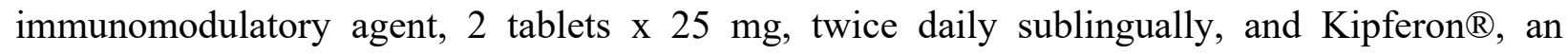
inducer of recombinant human interferon- $\alpha 2,1$ suppository twice daily.

All study subjects were tested weekly for SARS-CoV-2 using the polymerase chain reaction (PCR) method. At Week 6, all employees were tested for specific IgG.

\section{RESULTS}

Intranasal administration of Aprotinin significantly reduced the SARS-CoV-2 RNA titer 3 days p.i. in nasal washes compared to the Control group (Figure 1B). The SARS-CoV-2 RNA was absent in lung tissues of 5 animals from Aprotinin group and significantly reduced in 3 other hamsters compared to the Control group (Figure 1A). It means that prophylactic treatment with Aprotinin prevented SARS-CoV-2 reproduction and distribution to the lungs. Some difference in the body weight of hamsters (Figure 1C) is not statistically significant and most likely is not related to the influence of Aprotinin.

The prospective study of Aprotinin prophylactic treatment in health care personnel from MSMU demonstrated that only 2 out of 30 workers (6.7\%) were infected with SARS-CoV-2. The disease progression was asymptomatic and was determined at the week 6 only using $\operatorname{IgG}$ analysis. 


\section{DISCUSSION}

Preventive IN administration of Aprotinin to the hamsters 1 hour before SARS-CoV-2 infection prevented its distribution to the lungs and significantly reduced SARS-CoV-2 RNA titer in nasal washes and lung tissues (Figure 1). This is in good agreement with results of Aprotinin administration to health care professionals in the Covid hospital of MSMU - only 2 $(6.7 \%)$ people out of 30 were infected and had mild severity of or no symptoms. At the same time, the average infection rate of health care professionals can reach $29 \%$ [14].

No adverse events were recorded in volunteers taking Aprotinin during the prospective study in the Covid hospital of MSMU. All these data give grounds for further study and authorization of Aprotinin as the product for pharmacological prevention of SARS-CoV-2 (COVID-19).

POTENTIAL CONFLICTS OF INTEREST. Preclinical studies were performed at the State Research Center of Virology and Biotechnology VECTOR. The clinical trial was by First Moscow State Medical University named I. M. Sechenov.

ACKNOWLEDGMENTS. The authors thank as well as members of the Independent Data Monitoring Committee, including MD, PhD, Professor Alexey V. Kravchenko of Central Research Institute of Epidemiology (Moscow, Russia), MD, PhD, Professor, Russian Academy of Sciences Professor Kirill A. Zykov of Moscow State Medical Dental University n.a. A.I. Yevdokimov (Moscow, Russia) and MD, PhD, Professor Vladimir V. Rafalskiy of Immanuel Kant Baltic Federal University (Kaliningrad, Russia).

FUNDING. The Ministry of Industry and Trade of the Russian Federation

\section{REFERENCES}

1. Russian Federation first registered vaccine from coronavirus. Interfax.RU August 112020. https://www.interfax.ru/news/2020/08/11.

https://sia.ru/?section=484\&action=show_news\&id=399342

2. Prevention and Prophylaxis of SARS-CoV-2 Infection. Last Updated: July 17, 2020. Available at: https://www.covid19treatmentguidelines.nih.gov/ overview/prevention-of-sars$\operatorname{cov}-2 /$. 
3. Bojkova D, McGreig JE, McLaughlin K-M, Masterson SG, Widera M, Krahling V, Ciesek S, Wass MN, Michaelis M, Cinati jr J. SARS-CoV-2 and SARS-CoV differ in their cell tropism and drug sensitivity profiles. BioRxiv, doi: https://doi.org/10.1101/2020.04.03.024257.

4. Bestle D, Heindl MR, Limburg $\mathrm{H}$, et al. TMPRSS2 and furin are both essential for proteolytic activation of SARS-CoV-2 in human airway cells. Life Sci Alliance. 2020;3(9):e202000786. Published 2020 Jul 23. doi:10.26508/1sa.202000786

5. Hoffmann M, Kleine-Weber H, Schroeder S, et al. SARS-CoV-2 Cell Entry Depends on ACE2 and TMPRSS2 and Is Blocked by a Clinically Proven Protease Inhibitor. Cell. 2020;181(2):271-280.e8. doi:10.1016/j.cell.2020.02.052

6. Bugge TH, Antalis TM, Wu Q. Type II transmembrane serine proteases. 2009. J Biol Chem 284:23177-81. Review.

7. Böttcher-Friebertshäuser E. 2018. Membrane-Anchored Serine Proteases: Host Cell Factors in Proteolytic Activation of Viral Glycoproteins. In: Böttcher-Friebertshäuser E., Garten W., Klenk H. (eds) Activation of Viruses by Host Proteases. Springer, Cham, p.153-203. Review

8. Hoffmann M, Hofmann-Winkler H, Pöhlmann S. 2018. Priming Time: How Cellular Proteases Arm Coronavirus Spike Proteins. In: Böttcher-Friebertshäuser E., Garten W., Klenk H. (eds) Activation of Viruses by Host Proteases. Springer, Cham, p.71-98. Review

9. Moggia E, Koti R, Belgaumkar AP, Fazio F, Pereira SP, Davidson BR, Gurusamy KS.Pharmacological interventions for acute pancreatitis. Cochrane Database Syst Rev.2017 Apr $21 ; 4: C D 011384$.

10. Kapadia BH, Torre BB, Ullman N, Yang A, Harb MA, Grieco PW, Newman JM, Harwin SF, Maheshwari AV. Reducing perioperative blood loss with antifibrinolytics and antifibrinolytic-like agents for patients undergoing total hip and total knee arthroplasty. Orthop. 2019 Jul 2;16(6):513-516.

11. Trasylol pachage insert 12/03 (C2003 Bayer Pharmaceuticals Corporation

12. O.P. Zhirnov, H. D. Klenk, P. F. Wright. Aprotinin and similar protease inhibitors as drugs against influenza. Antiviral. Res. 2011, 92(1), 27-36. doi: 10.1016/j.antiviral.2011.07.014

13. O. P. Zhirnov, P. B. Golyando, A. V. Ovcharenko. Replication of Influenza B virus in chicken embryos is suppressed by exogenous aprotinin. Archives of Virology 1994, 135, 209216. https://doi.org/10.1007/BF01309780

14. Ovcharenko, A. V., \& Zhirnov, O. P. (1994). Aprotinin aerosol treatment of influenza and paramyxovirus bronchopneumonia of mice. Antiviral research, 23(2), 107-118. https://doi.org/10.1016/0166-3542(94)90038-8. 
15. Ovcharenko, A. V., \& Zhirnov, O. P. (1994). Aprotinin aerosol treatment of influenza and paramyxovirus bronchopneumonia of mice. Antiviral research, 23(2), 107-118. https://doi.org/10.1016/0166-3542(94)90038-8.

16. Shen LW, Mao HJ, Wu YL, Tanaka Y, Zhang W. TMPRSS2: A potential target for treatment of influenza virus and coronavirus infections. Biochimie. 2017 Nov;142:1-10.

17. Medical prophylaxis of COVID-19 in adults. Temporary recommendations. Prophylaxis, Diagnosis and Therapy of Novel Coronavirus Infection (COVID-19). Ministry of Health Russian Federation, Version 7, p.71 (03.06.2020).

https://static-0.rosminzdrav.ru/system/attachments/attaches/000/050/584/original/

03062020_\%D0\%9CR_COVID-19_v7.pdf.

\section{Figure 1}

Prophylactic efficacy of aprotinin on morbidity of SARS-CoV-2 virus-infected hamsters. Hamsters ( $n=8$ /group) were lightly anesthetized with ether, and aprotinin (1000 KIU/animal) was administered intranasally twice daily for three days. The control (untreated) animals received sodium saline on the same schedule. One hour after the first aprotinin dose, each hamster was inoculated with $10^{3} \mathrm{CPE}_{50}$ of SARS-CoV-2 (100 $\mu \mathrm{l} /$ animal). Virus replication from samples obtained from lung tissues (A) and nasal washes (B) of hamsters at 3 day p.i. was determined by qRT-PCR (limit of detection, $\mathrm{Ct}=40$ ). Weight changes in body weight of hamsters were determined at 3 day p.i. (C). $* P<0.05$ relative to control group (t-test).

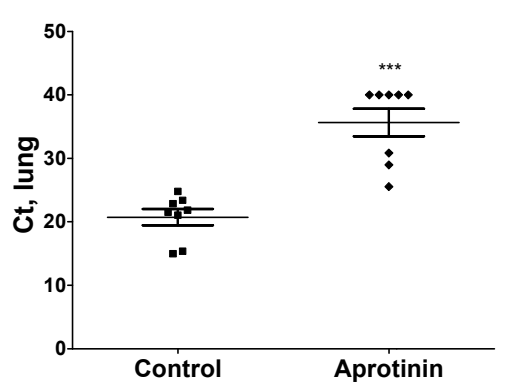

A

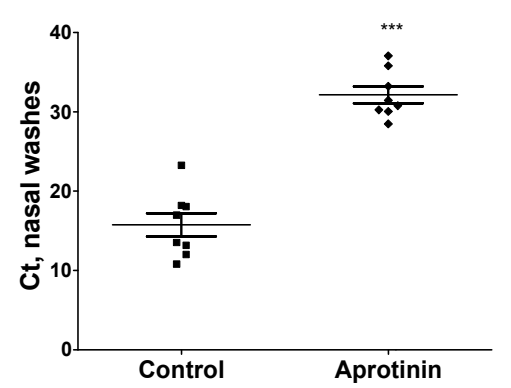

B

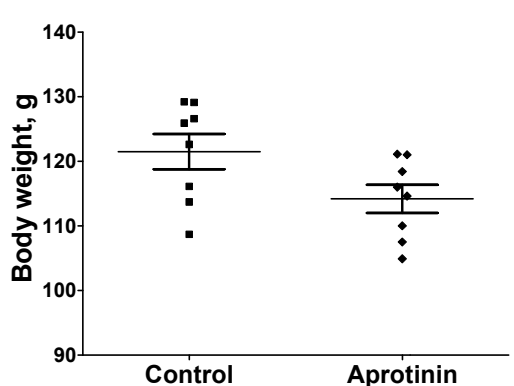

$\mathrm{C}$ 